\title{
Experimental investigation on the field-dependent properties of magnetorheological elastomer with circular honeycomb holes
}

\author{
Miao Yu ${ }^{1,2}{ }^{*}$, Zhiwei Xing ${ }^{1}$, Xing Zheng ${ }^{1}$, Jie Fu ${ }^{1,2}$ and Seung-Bok Choi ${ }^{3}$ \\ College of Optoelectronic Engineering, Chongqing University, Chongqing, China \\ ${ }^{2}$ Key Laboratory for Optoelectronic Technology and Systems of the Ministry of Education, Chongqing University, Chongqing, China \\ ${ }^{3}$ Smart Structures and Systems Laboratory, Department of Mechanical Engineering, Inha University, Incheon, South Korea
}

\section{Edited by:}

Weihua Li, University of Wollongong, Australia

Reviewed by:

X. M. Zhang, Tohoku University, Japan Dmitry Borin, Dresden University of

Technology, Germany

*Correspondence:

Miao Yu, College of Optoelectronic

Engineering, Chongqing University,

Chongqing, 400044, China

e-mail:yumiao@cqu.edu.cn

In order to enhance adjustable mechanical properties of a specific magnetorheological elastomer (MRE), this study presents a new exterior structure of MRE by punching circular honeycomb holes on the MRE samples. Seven silicone rubber MRE samples with the same component are fabricated and then punched holes with different numbers and diameters. The influence of different porosities on the mechanical properties of MRE under various magnetic fields is experimentally investigated by using a rheometer with electromagnetic suite. It is shown from experimental investigation that the porosity of MRE samples has a significant impact on the performance of MRE; the shear storage modulus (MR effect), and the loss factor is greatly increased. It is also observed that all the field-induced mechanical properties of the samples attain their respective maximum performance when the porosity increases to a critical value. The experimental results presented in this work directly indicate that high performances of the field-dependent mechanical and rheological properties can be achieved by means of external alternative structures such as honeycomb holes.

Keywords: magnetorheological elastomer, mechanical property, rheological property, honeycomb holes, ordinary silicon rubber

\section{INTRODUCTION}

Magnetorheological elastomer (MRE) is a relatively new branch of magnetorheological (MR) material family, whose rheological, mechanical, and magnetic properties can be varied continuously, rapidly, and reversibly under applied magnetic field (Jolly et al., 1996; Gong et al., 2005). MRE mainly consists of soft magnetic particles in a rubber-like non-magnetic matrix material. The outstanding field-control characteristic means that MRE has wide application prospects in smart isolator (Opie and Yim, 2011; Li et al., 2012, 2013), adaptive absorber (Deng et al., 2006), and controllable actuator (Böse et al., 2012). Simultaneously, it is well known that the compromise between mechanical properties and MR effects of existing MRE materials limits their engineering applications in which suitable quasi-static and dynamic mechanics performances are coordinately demanded. In order to develop high-performance MRE, many researchers and investigators improved formula, raw material selection, as well as manufacturing process (Hu et al., 2005; Böse, 2007; Chen et al., 2007; Li et al., 2008; Ge et al., 2013). However, a few academics have studied the approaches of altering exterior structure of MRE to improve the mechanical performance of MRE. In the literature, York et al. (2007) designed MR fluid-elastomer (MRF-E) composite through encapsulating MR fluids into an elastomer to achieve both the stiffness and damping capability controlled by an external magnetic field. Zhang et al. (2008) fabricated the patterned MREs with predesigned lattice and body centered cubic (BCC) structures. Zhang et al. (2010) prepared a type of hybrid MRE, in which MRFs and magnetorheological gels (MRGs) were injected into the punched holes, to improve mechanical performance. Ju et al. (2012) fabricated isotropic porous MRE by using the decomposition reaction of filled $\mathrm{NH}_{4} \mathrm{HCO}_{3}$ to generate the porous structure. They showed that both MR effect and damping property of the porous MRE reached a higher level than that of the MRE without pores.

To this end, generally, circular cell honeycomb solids provide an alternative exterior structure with the potential for improved mechanical properties, which have small solid volume fractions or relative densities. Furthermore, the reasonable place to start this study is to investigate the influence of circular honeycomb structure in the field-dependent performance without changing the formula and process of the original MRE. Consequently, a technical novelty of this work is to propose a new silicone rubber MRE with circular honeycomb holes. In order to validate high performances of the proposed MRE, several MRE samples with different porosities are tested under shear mode in an increasing magnetic field. The dynamic magnetic-controllable mechanical properties of MRE such as shear storage modulus, MR effect, and loss factor are confirmed by calculation and analysis of experimental results. It is reasonably addressed that this study can propose an effective approach to improve the mechanical properties of MRE by changing external alternative structures without modification of MRE formula.

\section{MRE SAMPLES AND TESTING SYSTEM}

Magnetorheological elastomer specimens were composed by magnetic particles in an elastic matrix. To prepare MREs, a two-component room temperature vulcanizing (RTV) silicone 


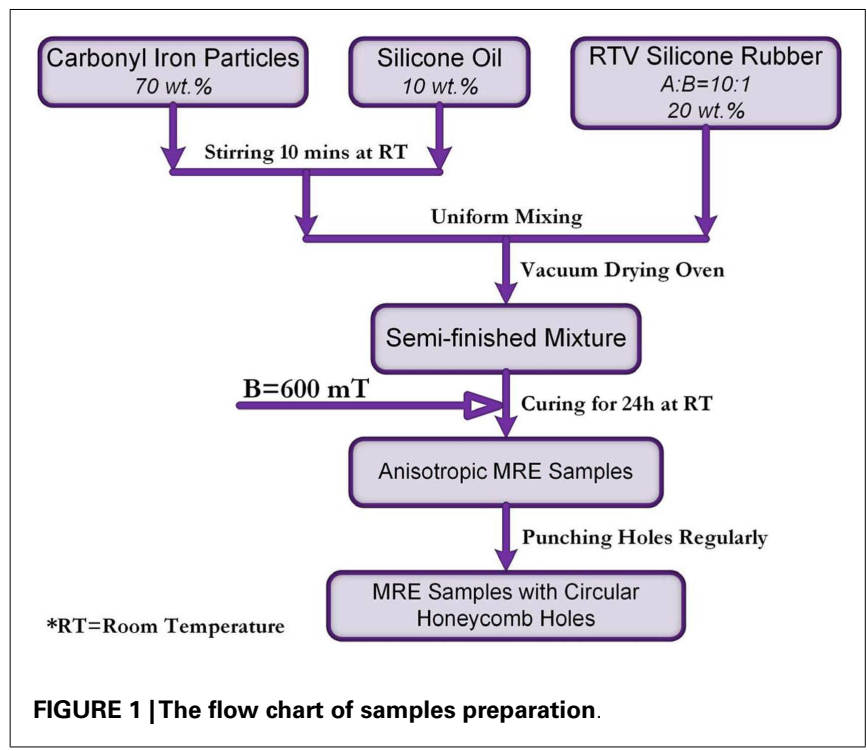

rubber (Type: SC-2110, Beijing Sanchen Industrial New Material Co. Ltd., China) was selected as the base elastomer materials, while a silicone oil with a kinetic viscosity of $500 \mathrm{cSt}$ was mixed within the specimen to change the ductility of the rubber base. The filler particles dispersed in the matrix were carbonyl iron particles (Type: JCF2-2, Jilin Jien Nickel Industry Co. Ltd., China) with the size distribution $d_{50}=5 \sim 8 \mu \mathrm{m}$. In all specimens, the mass fraction of the carbonyl iron particles, silicone rubber, and silicone oil was 70,20 , and 10\%, respectively. A flow chart of MRE samples preparation was shown in Figure 1. The fabrication procedure of MREs was as follows: firstly, the iron particles were mixed into the silicone rubber in a beaker and then stirred for about $10 \mathrm{~min}$ at room temperature. Secondly, after all ingredients were evenly mixed, the resulting material was put in a vacuum drying oven to remove air bubbles and then packed into an aluminum mold. Finally, the mixture was cured for $24 \mathrm{~h}$ at room temperature in a self-developed permanent magnet device with the magnetic field of $600 \mathrm{mT}$.

In this study, seven anisotropic MRE samples with the uniform size of $20 \mathrm{~mm}$ in diameter and $1 \mathrm{~mm}$ in thickness were cut from the prepared MRE specimens. Different numbers and sizes of holes were punched regularly in the MRE samples by using the punch with a suitable drill, as shown in Table 1. Moreover, the holes positioned parallel to the direction of the chain-like structure in the MREs. Figure $\mathbf{2}$ is the photograph of samples used in this experimental study.

Dynamic mechanical properties of MRE specimens were measured by using an advanced commercial rheometer (MCR 301, Anton Paar Co., Austria). The rheometer and its operating principle were shown in Figure 3. A parallel-plate rotor and a Magneto Rheological Device (MRD 170, Anton Paar Co., Austria) were installed in the rheometer. The diameter of the plate was $20 \mathrm{~mm}$ and the parallel-plate gap was fixed at $1 \mathrm{~mm}$. The testing magnetic field perpendicular to the direction of the shear flow was generated by adjusting the current value supplied to the electromagnetic coil, and testing temperatures were controlled by a water bath. A

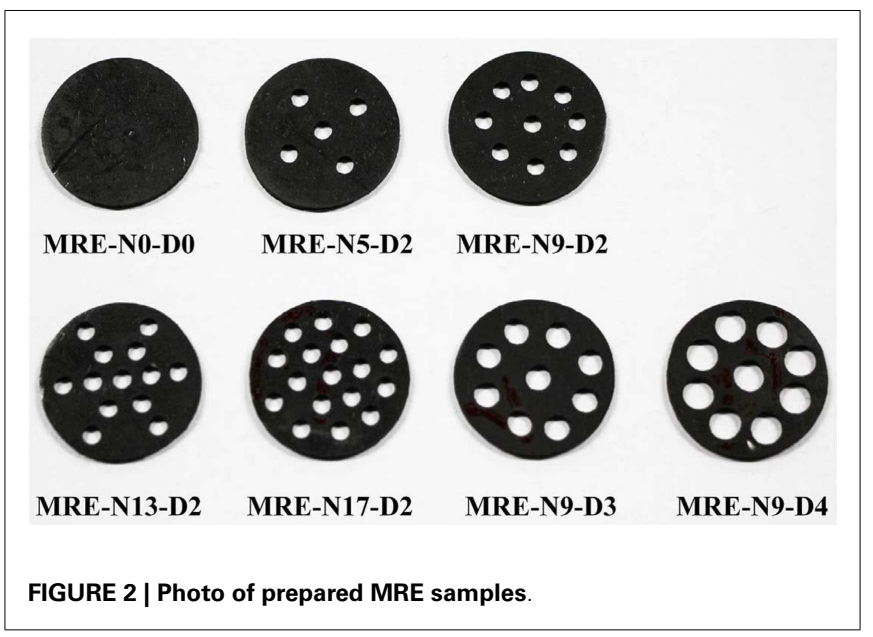

Table 1 | Specification of the prepared MRE samples.

\begin{tabular}{lccc}
\hline Sample name & $\begin{array}{c}\text { Hole } \\
\text { number }\end{array}$ & $\begin{array}{c}\text { Hole } \\
\text { diameter (mm) }\end{array}$ & Porosity (\%) \\
\hline MRE-N0-D0 & 0 & 0 & 0 \\
MRE-N5-D2 & 5 & 2 & 5.00 \\
MRE-N9-D2 & 9 & 2 & 9.00 \\
MRE-N13-D2 & 13 & 2 & 13.00 \\
MRE-N17-D2 & 17 & 2 & 17.00 \\
MRE-N9-D3 & 9 & 3 & 20.25 \\
MRE-N9-D4 & 9 & 4 & 36.00 \\
\hline
\end{tabular}

sample was placed between the rotating disk and the base without slip effect. When the rotating disk rotated, the sample worked in oscillation shear mode, in which the direction of the oscillation was represented by green arrow. This system applied a desired oscillatory strain amplitude and frequency to the sample and measured the amplitude and phase of the output force, from which the shear storage modulus and loss modulus were calculated. In the experiment, the MRE sample was sandwiched between rotating disk and base in parallel, in which the particle chains were distributed along the thickness direction. MRE samples were tested under shear mode at room temperature controlled by a water circulator. The shear oscillation frequency and shear strain amplitude were set as a constant $10 \mathrm{~Hz}$ and $1 \%$, respectively. To avoid the sliding effect between the sample and the parallel plate, a constant normal force of $10 \mathrm{~N}$ was applied during the tests. As shown in Figure 4, the shear stress appears to be a linear relationship with the increasing shear strain amplitudes ranging from 0.1 to $10 \%$ at $10 \mathrm{~Hz}$ during the previous dynamic strain sweep experiments without magnetic field, in which demonstrated that there is no sliding effect in the rheometer testing system. In addition, each sample was measured for three times at the same condition with the magnetic flux density changing from 0 to $1.2 \mathrm{~T}$.

\section{RESULTS AND DISCUSSIONS STORAGE MODULUS AND MR EFFECT}

The shear storage modulus $G^{\prime}$ for the punched MRE samples with different numbers of holes (different porosities) is shown 


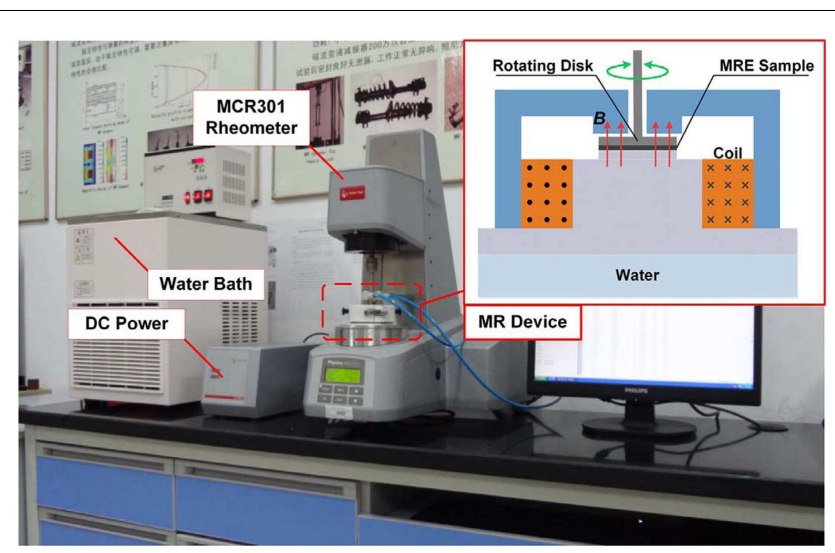

FIGURE 3 | Configuration of testing system

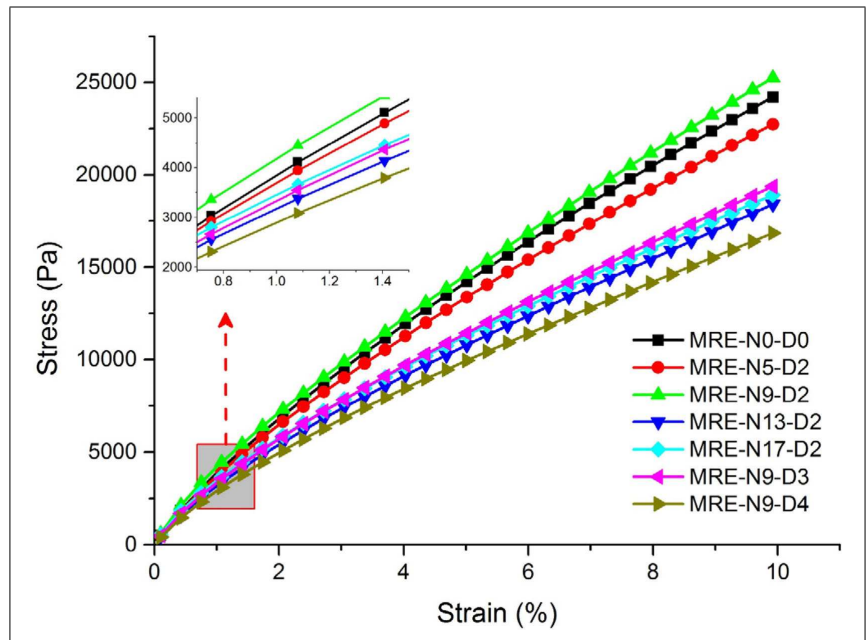

FIGURE 4 | Stress-strain curve of samples without magnetic field at $10 \mathrm{~Hz}$.

in Figure 5. As can be seen from the experimental data, the field-dependent storage modulus $G^{\prime}$ of each samples exhibit an increasing trend with increasing magnetic flux density, which was because the interaction among iron particles filled in the matrix resulted in an increase in mechanical stiffness. As shown in Figure 6, the zero-field storage modulus of non-porous MRE sample is larger than that of porous MRE samples. This is due to the integrity of sample. However, the modulus of the samples firstly increases with increasing porosity up to $9.00 \%$ (Sample MRE-N9-D2) and then modulus decreases with increasing porosity. A linear fitting results for the initial storage modulus and Gaussian fitting for the maximum modulus at magnetic saturation are presented in Figure 6. It can be easily seen that the field-induced storage modulus is reached a maximum of 1.3 $\mathrm{MPa}$ when the porosity is $9.00 \%$. With the increase of porosity, the shear storage modulus at magnetic saturation showed greater than that of non-porous MRE, as the chain-like structures of iron particles highly reduced. The MR effect is a critical

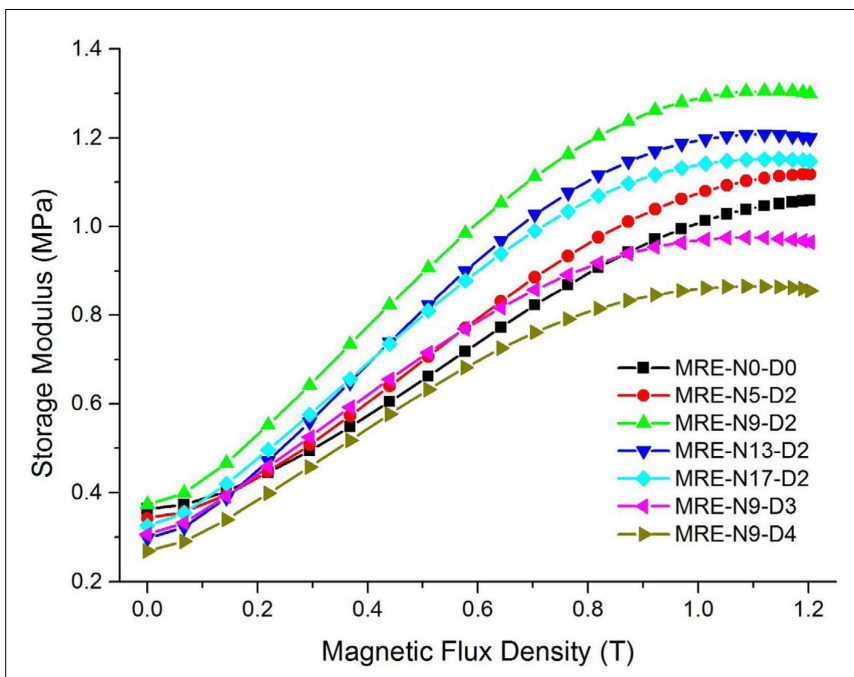

FIGURE 5 | Shear storage modulus of MRE samples under different magnetic fields.

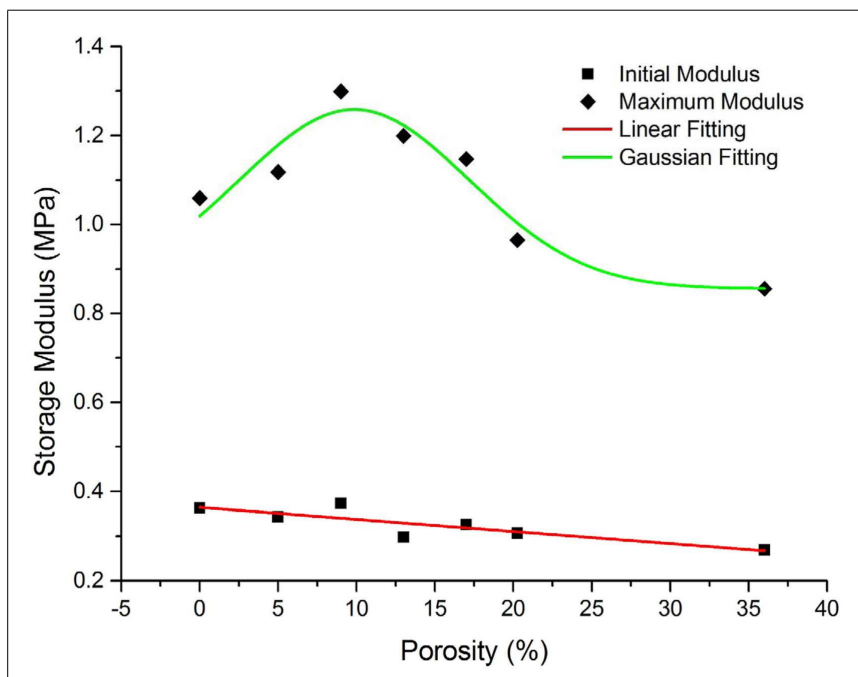

FIGURE 6 | Initial storage modulus $G_{0}$ and maximum storage modulus G_max' for different porosities.

parameter for evaluating MRE performance, and is defined as follow:

$$
G_{\mathrm{MR}-\mathrm{effect}}=100 \% \times\left(G_{\max }^{\prime}-G_{0}\right) / G_{0}
$$

where $G_{\max }^{\prime}$ is the shear storage at magnetic saturation, $G_{0}$ is the initial modulus. Figure 7 shows the relation between the MR effects and different porosities, in which the red curve has been given with a feasible Gaussian peak fitting. Obviously, the MR effects for the prepared samples reached a maximum of $300 \%$ when the porosity of MRE samples was $13.00 \%$ (Sample MRE-N13-D2). Due to a lower initial modulus, the MR effect of MRE-N13-D2 was higher than that of MRE-N9-D2, which had the largest magnetic modulus. As a result, it is obvious that MR effects 


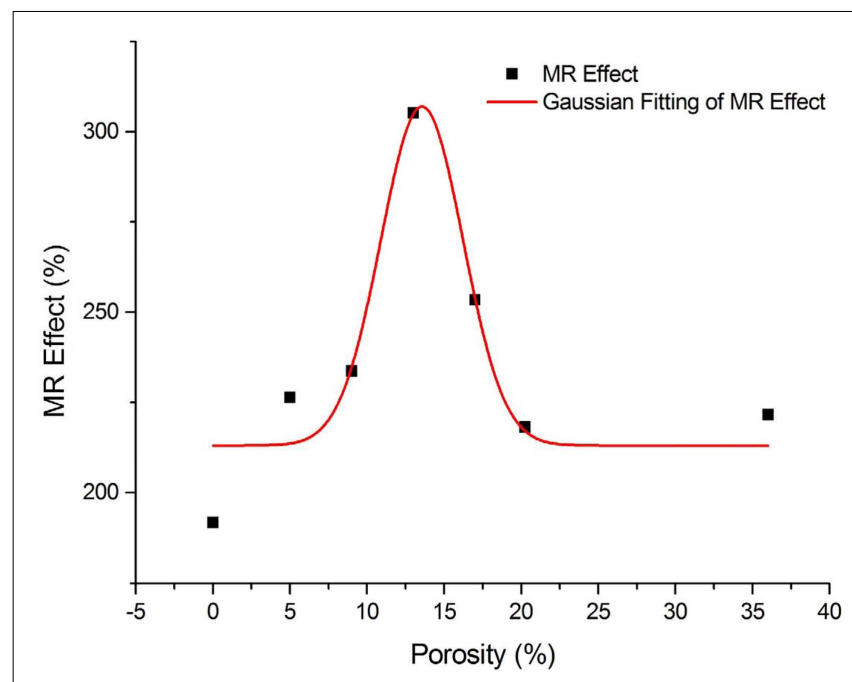

FIGURE 7 | MR effect of MRE samples with different porosities.

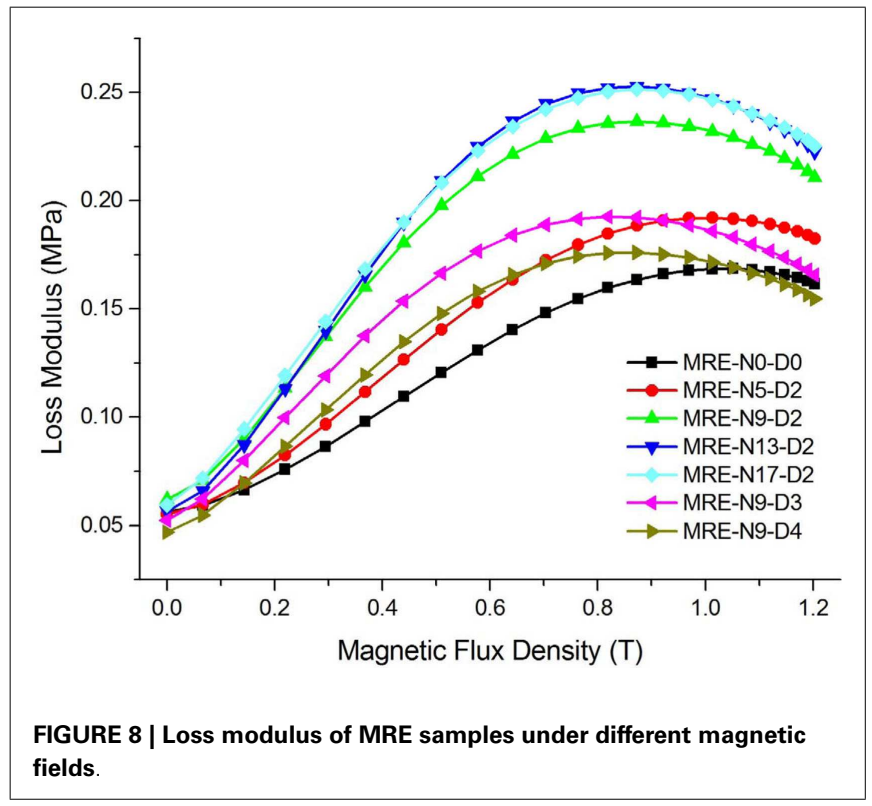

can be dramatically improved by punching circular honeycomb holes.

\section{LOSS MODULUS AND LOSS FACTOR}

The damping property of MRE with circular honeycomb holes is also investigated. The loss factor is an important factor of damping capability expressed as follows:

$$
\tan \delta=G^{\prime \prime} / G^{\prime}
$$

where $G^{\prime \prime}$ is the loss modulus. And to be clear, in the measurement of viscoelastic material, the loss modulus represents the level of dissipating energy by the viscous portion while the loss factor is

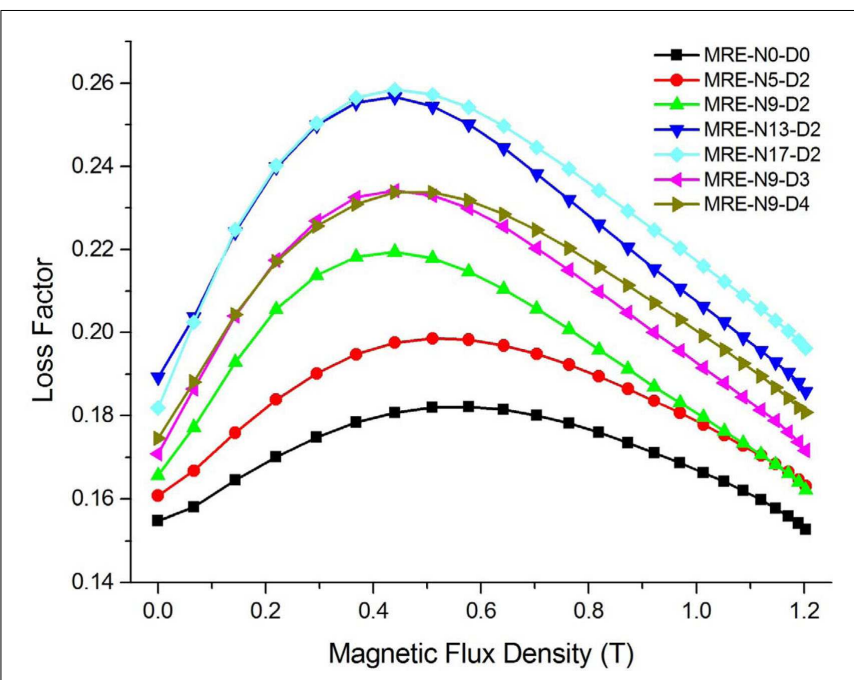

FIGURE 9 | Loss factor of MRE samples under different magnetic fields.

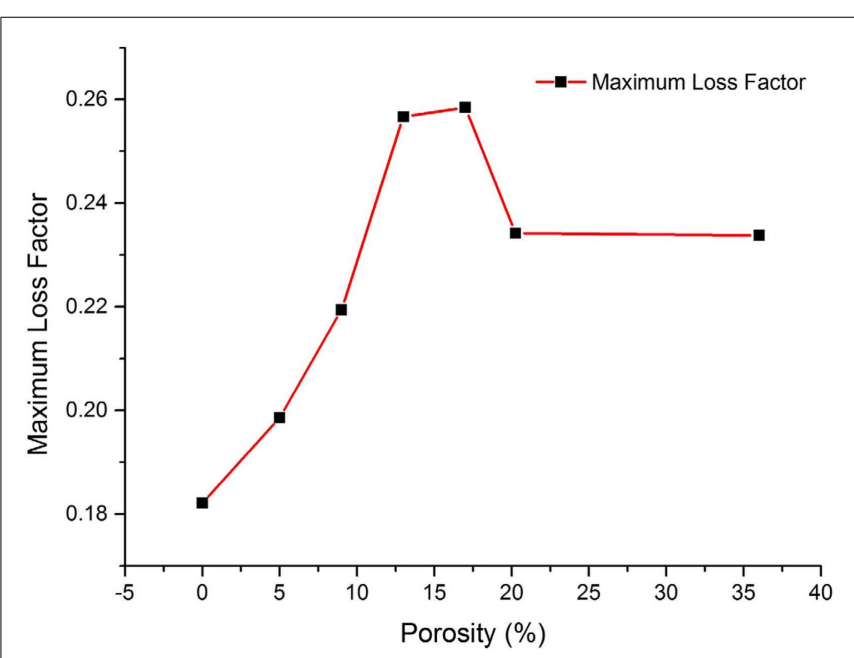

FIGURE 10 | Maximum loss factor for different porosities under magnetic field.

the ratio of loss modulus to storage modulus. Figures $\mathbf{8}$ and $\mathbf{9}$ show the loss modulus and loss factor of MRE samples with different porosities under different magnetic flux densities. It can be seen that both the loss modulus and the loss factor of punched MRE samples are higher than that of un-punched MRE sample to a variable extent. Furthermore, the two damping parameters have showed firstly increasing and then decreasing trend with the increasing porosity. It is seen from Figure 10 that the sample MREN13-D2 with a porosity of $13 \%$ and the sample MRE-N17-D2 with a porosity of $17 \%$ are reached to the best damping capacity. Due to the exterior structural changes after punching holes on the MRE samples, the damping property of MRE improved under various magnetic fields. 


\section{CONCLUSION}

In this work, a series of MREs with different numbers of twodimensional circular honeycomb holes were developed based on the ordinary silicon rubber MREs. The influence of circular honeycomb holes or porosities on the mechanical properties of MRE under different magnetic fields was experimentally investigated. The dynamic mechanical properties including storage modulus, MR effect, loss modulus, and loss factor have been significantly improved by punching holes on the MRE samples, which adopted various exterior structure of MRE samples. It has been also observed that the field-induced mechanical performance increases first and then decreases with the increasing porosity. The shear storage modulus, MR effect, loss modulus, and loss factor reached a maximum when the porosity of MRE samples were 9.00, 13.00, 13.00 , and $17.00 \%$, respectively. The experimental results presented in this work indicate that high-performance properties of MRE can be achieved without changing its preparation in the specific formula. In addition, this work can be a useful guideline to choose optimal number of porosity, which can produce maximum performances of mechanical and rheological properties of MRE under the same magnetic field.

\section{ACKNOWLEDGMENTS}

This work was financially supported by the National Natural Science Foundation of China (Grant No. 61203098), the National Science and Technology Support Program (2012BAF06B00F).

\section{REFERENCES}

Böse, H. (2007). Viscoelastic properties of silicone-based magnetorheological elastomers. Int. J. Mod. Phys. B 21, 4790-4797. doi:10.1142/S0217979207045670

Böse, H., Rabindranath, R., and Ehrlich, J. (2012). Soft magnetorheological elastomers as new actuators for valves. J. Intell. Mater. Syst. Struct. 23, 989-994. doi:10.1177/1045389X11433498

Chen, L., Gong, X. L., Jiang, W. Q., Yao, J. J., Deng, H. X., and Li, W. H. (2007). Investigation on magnetorheological elastomers based on natural rubber. J. Sci. Mater. 42, 5483-5489. doi:10.1007/s10853-006-0975-x

Deng, H. X., Gong, X. L., and Wang, L. H. (2006). Development of an adaptive tuned vibration absorber with magnetorheological elastomer. Smart Mater. Struct. 15, N111-N116. doi:10.1088/0964-1726/15/5/N02

Ge, L., Gong, X., Fan, Y., and Xuan, S. (2013). Preparation and mechanical properties of the magnetorheological elastomer based on natural rubber/rosin glycerin hybrid matrix. Smart Mater. Struct. 22, 115029. doi:10.1088/0964-1726/22/11/ 115029

Gong, X. L., Zhang, X. Z., and Zhang, P. Q. (2005). Fabrication and characterization of isotropic magnetorheological elastomers. Polym. Test. 24, 669-676. doi:10.1016/j.polymertesting.2005.03.015
Hu, Y., Wang, Y. L., Gong, X. L., Gong, X. Q., Zhang, X. Z., Jiang, W. Q., et al. (2005). New magnetorheological elastomers based on polyurethane/Sirubber hybrid. Polym. Test. 24, 324-329. doi:10.1016/j.polymertesting.2004.11. 003

Jolly, M. R., Carlson, J. D., and Muñoz, B. C. (1996). A model of the behaviour of magnetorheological materials. Smart Mater. Struct. 5, 607-614. doi:10.1088/ 0964-1726/5/5/009

Ju, B. X., Yu, M., Fu, J., Yang, Q., Liu, X. Q., and Zheng, X. (2012). A novel porous magnetorheological elastomer: preparation and evaluation. Smart Mater. Struct. 21, 035001. doi:10.1088/0964-1726/21/3/035001

Li, J. F., Gong, X. L., Xu, Z. B., and Jiang, W. Q. (2008). The effect of pre-structure process on magnetorheological elastomer performance. Int. J. Mater. Res. 99, 1358-1364. doi:10.3139/146.101775

Li, W. H., Zhang, X. Z., and Du, H. P. (2012). Development and simulation evaluation of a magnetorheological elastomer isolator for seat vibration control. J. Intell. Mater. Syst. Struct. 23, 1041-1048. doi:10.1177/1045389x11435431

Li, Y., Li, J., Li, W., and Samali, B. (2013). Development and characterization of a magnetorheological elastomer based adaptive seismic isolator. Smart Mater. Struct. 22, 035005. doi:10.1088/0964-1726/22/3/035005

Opie, S., and Yim, W. (2011). Design and control of a real-time variable modulus vibration isolator. J. Intell. Mater. Syst. Struct. 22, 113-125. doi:10.1177/ 1045389X10389204

York, D., Wang, X., and Gordaninejad, F. (2007). A new MR fluid-elastomer vibration isolator. J. Intell. Mater. Syst. Struct. 18, 1221-1225. doi:10.1177/ 1045389X07083622

Zhang, W., Gong, X. L., Xuan, S. H., and Xu, Y. G. (2010). High-performance hybrid magnetorheological materials: preparation and mechanical properties. Ind. Eng. Chem. Res. 49, 12471-12476. doi:10.1021/ie101904f

Zhang, X., Peng, S., Wen, W., and Li, W. (2008). Analysis and fabrication of patterned magnetorheological elastomers. Smart Mater. Struct. 17, 045001. doi:10.1088/0964-1726/17/4/045001

Conflict of Interest Statement: The authors declare that the research was conducted in the absence of any commercial or financial relationships that could be construed as a potential conflict of interest.

Received: 02 September 2014; accepted: 11 December 2014; published online: 05 January 2015.

Citation: Yu M, Xing Z, Zheng X, Fu J and Choi S-B (2015) Experimental investigation on the field-dependent properties of magnetorheological elastomer with circular honeycomb holes. Front. Mater. 1:34. doi: 10.3389/fmats.2014.00034

This article was submitted to Smart Materials, a section of the journal Frontiers in Materials.

Copyright (C) $2015 \mathrm{Yu}$, Xing, Zheng, Fu and Choi. This is an open-access article distributed under the terms of the Creative Commons Attribution License (CC BY). The use, distribution or reproduction in other forums is permitted, provided the original author(s) or licensor are credited and that the original publication in this journal is cited, in accordance with accepted academic practice. No use, distribution or reproduction is permitted which does not comply with these terms. 